\author{
Material Science
}

\title{
Effect of Rare Earth Doping on the Optical Quality of $A^{2} B^{6}$ Semiconductors
}

\author{
Yuriy P Gnatenko*, Anatoliy P Bukivskii and Petro M Bukivskij \\ Institute of Physics of NASU, Ukraine
}

*Corresponding author: Yuriy P Gnatenko, Department of Optics and Spectroscopy of

Crystals, Institute of Physics of NASU, Ukraine.

Received Date: March 01, 2019

Published Date: March 12, 2019

\section{Introduction}

It is well known that rare earth elements (RE) have a characteristic configuration of their electron shell, namely, $4 d^{10} 4 f^{n} 5 s^{2} 5 p^{6} 6 s^{2}(n=1-14)$, where $4 f$-electrons form an unfilled electronic shell shielded by several filled shells [1,2]. Thus, doping of semiconductor materials with RE leads to the appearance of a system of discrete energy levels associated with the ground and excited states of RE ions. These states are strongly localized and weakly perceive the vibrations of the crystalline lattice. Therefore, the emission of RE ions, caused by optical transitions from excited to ground states of the ions, includes narrow lines that are commonly used for developing laser systems [3]. In addition, the development of LEDs for a wide spectral region requires using $\mathrm{A}^{3} \mathrm{~B}^{5}$ semiconductors with wide band gaps doped with triply-charged RE ions. In this case, isovalent substitution of cations occurs.

Doping of semiconductors with $\mathrm{RE}$ ions is also used in semiconductor technology in order to reduce the concentration of residual impurities and other defects that strongly affect their electronic properties, decreasing the lifetime and the diffusion length of photogenerated carriers [4]. Introduction of RE ions allows us to clean the semiconductor materials from the residual impurities as a result of the manifestation of the gettering effect [5]. The point is that RE ions react strongly with metals and other atoms at a high temperature, forming neutral compounds that are removed from the the volume of the crystal during crystal growth.

Currently, semiconductors of $\mathrm{A}^{2} \mathrm{~B}^{6}$ group are widely used for the development of various devices of modern optoelectronics, in particular, solar cells, radiation detectors etc [6]. The effectiveness of these materials largely depends on their optical and crystalline quality and, accordingly, on the defective structure of the crystals, which determines their transport properties. However, there is a certain complexity associated with doping $\mathrm{A}^{2} \mathrm{~B}^{6}$ semiconductors with REs, since their solubility for such semiconductors is limited. In addition, here it is necessary to substitute the doubly-charged cationic sites with triply-charged RE ions. Thus, there are only a small number of works devoted to the effect of RE ions on the optical and crystal quality of $\mathrm{A}^{2} \mathrm{~B}^{6}$ semiconductors.

In this paper, we present the results of a comprehensive study of some single crystals and polycrystalline thin films of $\mathrm{A}^{2} \mathrm{~B}^{6}$ semiconductors, devoted to improving their optical and structural quality by doping with rare earth elements.

\section{Excitons as Nanosized Probes of a Crystal Structure}

It should be noted that optical research is an effective technique which makes it possible to obtain information on the optical and crystal quality of semiconductor materials. In this case, the exciton, that is, a coupled electron-hole pair, is used as a nano-probe of the crystal lattice heterogeneity [7]. The exciton radius for $\mathrm{A}^{2} \mathrm{~B}^{6}$ semiconductors corresponds to several $\mathrm{nm}$. It is well known that free excitons are manifested for semiconductor single crystals of high optical quality in the exciton absorption and reflection spectra. In addition, these excitons can also be detected in the photoluminescence (PL) spectra. The intensity of free excitons in the PL spectra is usually low, since the most intense PL lines in the exciton region correspond to bound excitons, i.e excitons bound with neutral acceptor ( $\mathrm{A}^{\circ} \mathrm{X}$-line) or donor $\left(\mathrm{D}^{\circ} \mathrm{X}\right.$-line) centers [8]. It should be noted that exciton lines usually manifest themselves in the optical spectra at low temperatures, since for many $\mathrm{A}^{2} \mathrm{~B}^{6}$ semiconductors the binding energy of free excitons is less than or equal to the thermal energy at $\mathrm{T}=300 \mathrm{~K}$ [9].

Thus, the analysis of the structure of low-temperature optical spectra allows us to obtain information on impurity and intrinsic defects, as well as on two-dimenshional dislocation-type defects for the studied semiconductor materials. 
The low temperature ( $\mathrm{T}=4.5 \mathrm{~K}$ ) PL spectra of CdTe single crystal doped with RE elements (Dy or Er) were studied in $[10,11]$. In particular, it was shown that the most intense PL line located in the short-wavelength spectral region corresponds to the $\mathrm{A}^{\circ} \mathrm{X}$-line associated with excitons bounded with Li or Na residual atoms. The bending on the short-wavelength edge of this line correponds to the emission of free excitons. The $\mathrm{A}^{\circ} \mathrm{X}$-line associated with a cadmium vacancy as a neutral acceptor was also observed. However, its intensity is very low, which indicates a low concentration of cadmium vacancies. It should be noted that the free excitons are also observed in the exciton reflection spectra as dispersive curves. Besides, the excited state $(\mathrm{n}=2)$ of excitons is also found in the reflection spectrum. This allows us to determine the binding energy for a free exciton. The exciton reflection spectra for CdTe: Er and CdTe: Dy crystals are very narrow $(\approx 1 \mathrm{meV})$. The exciton absorption spectra for bulk semiconductors are not observed, since the maximum absorption coefficient for a free exciton is approximately $10^{5} \mathrm{~cm}^{-1}$. To observe the exciton absorption spectra it is necessary to have crystal samples about 1 micron thick.

Thus, the optical research of CdTe crystals doped with Er or Dy shows their excellent optical quality. This is primarily due to the effect of gettering associated with RE, which form neutral compounds with many residual impurities ( $\mathrm{Ag}, \mathrm{Cl}, \mathrm{Si}, \mathrm{Cu}$, etc). For CdTe-based solid solutions (CdZnTe, CdMnTe, CdMgTe), an important intrinsic defect is associated with the Te precipitates, which strongly influences transport properties of the crystals by degrading their parameters (mobilities and lifetimes of carriers) [12]. In this case, the neutral $\mathrm{Dy}_{2} \mathrm{Te}_{3}$ compound is formed. Since the triply-charged RE ions substitute the double-charged cations, they act as donor atoms. Thus, the doping of $\mathrm{A}^{2} \mathrm{~B}^{6}$ semiconductors induces free electrons, which to some extent can compensate for acceptors. It should be noted that they also strongly influence other intrinsic defects, primarily cadmium vacancies. Since RE elements substitute the cations, they highly reduce the concentration of cadmium vacancies, which are the main intrinsic defects in CdTe of p-type. Decreasing of these defects contributes both to improving the optical quality of semiconductor materials, and to obtaining high-resistivity crystals or semi-insulating crystals, which is essential for the development of high-performance radiation detectors.

It is known that the concentration of acceptors associated with cadmium vacancies for p-type CdTe crystals corresponds to about $0.1 \%$. Therefore, the concentration of RE elements should not exceed this value. In some works, doping of $\mathrm{A}^{2} \mathrm{~B}^{6}$ semiconductor materials with RE corresponds to several percent [13,14]. In this case, the gettering effect takes place, but, in addition, the RE precipitates form in the crystal. So, for such crystals, there are broad defect PL bands associated with the recombination of donoracceptor pairs involving the acceptor complexes which consist of cadmium vacancies and ionized donor. These acceptor complexes are usually formed in the crystal region where the dislocations occur. Thus, the optical quality of crystals doped with RE in high concentrations (several percent) deteriorates.
It is very important to study the effect of RE elements on the optical and crystal structures of thin semiconductor films. Such studies were performed in the works $[9,15,16]$. Since these films are polycrystalline, they are highly heterogeneous. Thus, first of all, it is necessary to optimize the technological conditions of film deposition. Further, the improvment of their crystal structure and optical properties can be achieved by doping with RE elements of low concentrations. In order to obtain more information on the change in crystal structure, it is also desirable to carry out structural measurements (SEM images and XRD-diffraction). Such studies were performed for the polycrystalline CdS: Dy thin films deposited by close-space vacuum sublimation method on the glass substrates [16].

It was shown that CdS: Dy thin films have a thickness of about 500nm and crystal grains from $0.1 \mu \mathrm{k}$ to $1 \mu \mathrm{k}$. They have a hexagonal crystalline structure, and the hexagonal axis $\mathrm{c}$ is oriented substantially perpendicular to the substrate plane. It was found that the optimal technological conditions of CdS: Dy films correspond to the substrate temperature $T_{s}=573 \mathrm{~K}$ and the evaporator temperature $T_{e}=1173 \mathrm{~K}$.

The study of the low-temperature PL spectra of CdS: Dy films shows that in the short-wavelength spectral region narrow exciton $\mathrm{A}^{\circ} \mathrm{X}$ and $\mathrm{D}^{\circ} \mathrm{X}$ lines are observed. In addition, the free exciton lines also appear in the reflection and absorption spectra. As far as we know, the presence of free excitons simultaneously in the photoluminescence, reflection and absorption spectra has not previously been observed for any semiconductor films obtained by various methods, including the method of molecular epitaxy.

\section{Conclusion}

The effect of doping with rare earth elements on the optical properties, as well as on the defect structure of bulk single crystals and thin polycrystalline films, was discussed. It was shown that this is due to a significant reduction in cadmium vacancies, complex acceptor centers, dislocations, and tellurium and sulfur precipitates. In addition, there is also a gettering effect of residual impurity atoms. The obtained results open the way to a breakthrough in the technology of producing thin polycrystalline films of high optical and crystalline quality based on $\mathrm{A}^{2} \mathrm{~B}^{6}$ semiconductors and in improving their transport characteristics. It is expected that the efficiency of optoelectronic devices developed on their basis should be significantly improved.

\section{Acknowledgement}

None

\section{Conflict of Interest}

No conflict of interest.

\section{References}

1. GH Diere, HM Crosswhite (1968) Spectra and Energy Levels of Rare Earth Ions in Crystals. Interscience Publishers, New York, USA, p. 401.

2. R Boyn (1988) 4f-4f Luminescence of Rare-Earth Centers in II-VI Compounds. Phys Stat Sol (b) 148(1): 11-47. 
3. L Kou, DC Hall, $\mathrm{H}$ Wu (1998) Room-temperature $1.5 \mu \mathrm{m}$ photoluminescence of Er3+-doped AlxGa1-xAs native oxides. Appl Phys Lett 72(26): 3411-3413.

4. NV Sochinskii, M Abellán, J Rodríguez-Fernández (2007) Effect of Yb concentration on the resistivity and lifetime of CdTe:Ge:Yb codoped crystals. Appl Phys Lett 91: 202112.

5. O Procházková, J Novotný, J Zavadil, K Žd'ánský (1999) Effect of rare earth addition on liquid phase epitaxial InP-based semiconductor layers. Materials Science and Engineering: B 66(1-3): 63-66.

6. R Triboulet, P Siffert (2010) CdTe and Related Compounds; Physics, Defects, Hetero- and Nano-structures, Crystal Growth, Surfaces and Applications. Physics, CdTe-based Nanostructures, CdTe-based Semimagnetic Semiconductors, Defects, Elsevier, Amsterdam, USA, p. 550.

7. Giuseppe Grosso, Giuseppe Pastori Parravicini (2014) Excitons, Plasmons, and Dielectric Screening in Crystals. Solid State Physics (Second Edition), Academic Press p. 287-331.

8. YuP Gnatenko, PM Bukivskij, IO Faryna, AS Opanasyuk, MM Ivashchenko (2014) Photoluminescence of high optical quality CdSe thinfilms depositedby close-spaced vacuum sublimation. J Lum 146: 174-177.

9. YuS Yeromenko, YuP Gnatenko, AS Opanasyuk, DI Kurbatov, PM Bukivskij, et al. (2018) Photoluminescence of high optical quality CdS: Dy thinfilms deposited byclose-spaced vacuum sublimation. J Lum 197: 343-348.
10. YuP Gnatenko, PM Bukivskij, AP Bukivskii, MS Furier (2018) Effect of Dy-doping on photoluminescence properties of CdTe crystals andtheir defect structure. Physica B: Condensed Matter 546: 89-92.

11. YuP Gnatenko, MS Furyer, AP Bukivskii, LM Tarakhan, RV Gamernyk (2015) Photoluminescence and photoelectric properties of CdTe crystalsdoped with Er atoms. J Lumin 160: 258-261.

12. R Gul, UN Roy, SU Egarievwe, E Bolotnikov, GS Camarda, et al. (2016) Point defects: Their influence on electron trapping, resistivity, and electron mobility-lifetime product in CdTexSe1-x detectors. Appl Phys Lett 119(2): 025702.

13. Tao Wang, Xin Ai, Ziang Yin, Qinghua Zhao, Boru Zhou, et al. (2019) Study on a co-doped CdZnTe crystal containing Yb and In. CrystEngComm (Advance Article)

14. C Jayachandraiah, K Siva Kumar, G Krishnaiah, N Madhusudhana Rao (2015) Influence of Dy dopant on structural and photoluminescence of Dy-doped ZnO nanoparticles. Journal of Alloys and Compounds 623 : 248-254.

15. YuP Gnatenko, PM Bukivskij, MS Furier, AP Bukivskii, AS Opanasyuk (2018) Temperature dependence of the band gap of high optical quality CdS:Dy thin films based on exciton spectra. Mater Res Express 5(12): 12590.

16. YuP Gnatenko, YuS Yeromenko, DI Kurbatov, PM Bukivskij, MS Furier, et al. (2018) Study of the Structural and Optical Properties of Dy Doped CdS Thin Films Deposited by Close-spaced Vacuum Sublimation. Journal of Nano- and Electronic Physics 10 (5): 05001. 